\title{
Batch, Off-policy and Model-free Apprenticeship Learning
}

\author{
Edouard Klein ${ }^{13}$, Matthieu Geist ${ }^{1}$, and Olivier Pietquin ${ }^{12}$ \\ 1. Supélec-Metz Campus, IMS Research group, France, prenom.nom@supelec.fr \\ 2. UMI 2958 CNRS - GeorgiaTech, France \\ 3. Equipe ABC, LORIA-CNRS, France.
}

\begin{abstract}
This paper addresses the problem of apprenticeship learning, that is learning control policies from demonstration by an expert. An efficient framework for it is inverse reinforcement learning (IRL). Based on the assumption that the expert maximizes a utility function, IRL aims at learning the underlying reward from example trajectories. Many IRL algorithms assume that the reward function is linearly parameterized and rely on the computation of some associated feature expectations, which is done through Monte Carlo simulation. However, this assumes to have full trajectories for the expert policy as well as at least a generative model for intermediate policies. In this paper, we introduce a temporal difference method, namely LSTD- $\mu$, to compute these feature expectations. This allows extending apprenticeship learning to a batch and off-policy setting.
\end{abstract}

\section{Introduction}

Optimal control consists in putting a machine in control of a system with the goal of fulfilling a specific task, optimality being defined as how well the task is performed. Various solutions to this problem exist from automation to planification. Notably, the reinforcement learning (RL) paradigm [Sutton and Barto, 1998] is a general machine learning framework in which an agent learns to control optimally a dynamic system through interactions with it. The task is specified through a reward function, the agent objective being to take sequential decisions so as to maximize the expected cumulative reward.

However, defining optimality (through the reward function) can itself be a challenge. If the system can be empirically controlled by an expert, even though his/her behavior can be difficult to describe formally, apprenticeship learning is a way to have a machine controlling the system by mimicking the expert. Rather than directly mimicking the expert with some supervised learning approach, Inverse Reinforcement Learning (IRL) [Ng and Russell, 2000] consists in learning a reward function under which the policy demonstrated by the expert is optimal. Mimicking the expert therefore ends up to learning an optimal policy according to this reward function. A significant advantage of such an approach is that expert's actions can be guessed in states which have not been encountered during demonstration. Firstly introduced in [Russell, 1998], another advantage claimed 
by the author would be to find a compact and complete representation of the task in the form of the reward function.

There roughly exists three families of IRL algorithms: feature-expectationbased [Abbeel and Ng, 2004; Syed et al., 2008; Syed and Schapire, 2008; Ziebart et al., 2008], margin-maximization-based [Ratliff et al., 2006, 2007a,b; Kolter et al., $2008]$ and approaches based on the parameterization of the policy by the reward function [Ramachandran and Amir, 2007; Neu and Szepesvári, 2007]. The first family assumes a linearly parameterized reward function. This naturally leads to a linearly parameterized value function, the associated feature vector being the so-called feature expectation (see Section 2 for a formal definition). These approaches learn a reward function such that the feature expectation of the optimal policy (according to the learnt reward function) is close to the feature expectation of the expert policy. This is a sufficient condition to have close value functions (for any parameterized reward function, and therefore particularly the optimized one). The second family expresses IRL as a constrained optimization problem in which expert's examples have higher expected cumulative reward than all other policies by a certain margin. Moreover, suboptimality of the expert can be considered through the introduction of slack variables. The last family parameterizes policies with a reward function. Assuming that the expert acts according to a Gibbs policy (respectively to the optimal value function related to the reward function which is optimized), it is possible to estimate the likelihood of a set of state-action pairs provided by the expert. The algorithms differ in the way this likelihood is maximized.

This paper focuses on the first family of algorithms, and more precisely on the seminal work of Abbeel and $\mathrm{Ng}$ [2004]. All of them rely on the computation of the feature expectation (which depends on policies but not on rewards) of (i) the expert and (ii) some intermediate policies. The expert's feature expectation is computed using a simple Monte Carlo approach (which requires full trajectories of the expert). Other feature expectations are either computed exactly (which requires knowing analytically the dynamics of the system) or with a Monte Carlo approach (which requires simulating the system). The contribution of this paper is LSTD- $\mu$, a new temporal-difference-based algorithm for estimating these feature expectations. It relaxes the preceding assumptions: transitions of the expert are sufficient (rather than full trajectories) and nor the model neither a simulator are necessary to compute intermediate feature expectations. This paper focuses on the algorithm introduced in [Abbeel and Ng, 2004], but the proposed approach can be used in other algorithms based on feature expectation computation.

The rest of this paper is organized as follows. Section 2 provides the necessary background, notably the definition of feature expectation and its use in the seminal IRL algorithm of Abbeel and Ng [2004]. Section 3 presents LSTD- $\mu$, our main contribution. Section 4 provides some preliminary experiments and Section 5 opens perspectives. 


\section{Background}

A sequential decision problem is often framed as a Markov Decision Process (MDP) [Sutton and Barto, 1998], a tuple $\{S, A, P, R, \gamma\}$ with $S$ being the state space, $A$ the action space, $P \in \mathcal{P}(S)^{S \times A}$ the set of Markovian transition probabilities, $R \in \mathbb{R}^{S}$ the reward function (assumed to be absolutely bounded by 1) and $\gamma \in\left[0,1\left[\right.\right.$ a discounting factor. A policy $\pi \in A^{S}$ maps states to actions. The quality of a policy is quantified by the associated value function $V^{\pi}$, which associates to each state the expected and discounted cumulative reward: $V^{\pi}(s)=E\left[\sum_{t=0}^{\infty} \gamma^{t} R\left(s_{t}\right) \mid s_{0}=s, \pi\right]$. Dynamic programming aims at finding the optimal policy $\pi^{*}$, that is one of the policies associated with the optimal value function, $V^{*}=\operatorname{argmax}_{\pi} V^{\pi}$, which maximizes the value for each state. If the model (that is transition probabilities and the reward function) is unknown, learning the optimal control through interactions is addressed by RL.

For IRL, the problem is reversed. It is assumed that an expert acts according to an optimal policy $\pi_{E}$, this policy being optimal according to some unknown reward function $R^{*}$. The goal is to learn this reward function from sampled trajectories of the expert. This is a difficult and ill-posed problem $[\mathrm{Ng}$ and Russell, 2000]. Apprenticeship learning through IRL, which is the problem at hand in this paper, has a somewhat weaker objective: it aims at learning a policy $\tilde{\pi}$ which is (approximately) as good as the expert policy $\pi_{E}$ under the unknown reward function $R^{*}$, for a known initial state $s_{0}$ (this condition can be easily weakened by assuming a distribution over initial states). Now, the approach proposed in [Abbeel and $\mathrm{Ng}, 2004$ ] is presented.

We assume that the true reward function belongs to some hypothesis space $\mathcal{H}_{\phi}=\left\{\theta^{T} \phi(s), \theta \in \mathbb{R}^{p}\right\}$, of which we assume the basis functions to be bounded by $1:\left|\phi_{i}(s)\right| \leq 1, \forall s \in S, 1 \leq i \leq p$. Therefore, there exists a parameter vector $\theta^{*}$ such that: $R^{*}(s)=\left(\theta^{*}\right)^{T} \phi(s)$ In order to ensure that rewards are bounded, we impose that $\|\theta\|_{2} \leq 1$. For any reward function belonging to $\mathcal{H}_{\phi}$ and for any policy $\pi$, the related value function $V^{\pi}(s)$ can be expressed as follows:

$$
V^{\pi}(s)=E\left[\sum_{t=0}^{\infty} \gamma^{t} \theta^{T} \phi\left(s_{t}\right) \mid s_{0}=s, \pi\right]=\theta^{T} E\left[\sum_{t=0}^{\infty} \gamma^{t} \phi\left(s_{t}\right) \mid s_{0}=s, \pi\right]
$$

Therefore, the value function is also linearly parameterized, with the same weights and with basis functions being grouped into the so-called feature expectation $\mu^{\pi}$ :

$$
\mu^{\pi}(s)=E\left[\sum_{t=0}^{\infty} \gamma^{t} \phi\left(s_{t}\right) \mid s_{0}=s, \pi\right]
$$

Recall that the problem is to find a policy whose performance is close to that of the expert's for the starting state $s_{0}$, on the unknown reward function $R^{*}$. In order to achieve this goal, it is proposed in [Abbeel and $\mathrm{Ng}, 2004$ ] to find a policy $\tilde{\pi}$ such that $\left\|\mu^{\pi_{E}}\left(s_{0}\right)-\mu^{\tilde{\pi}}\left(s_{0}\right)\right\|_{2} \leq \epsilon$ for some (small) $\epsilon>0$. Actually, this ensures that the value of the expert's policy and the value of the estimated policy (for the starting state $s_{0}$ ) are close for any reward function of $\mathcal{H}_{\phi}$ :

$$
\left|V^{\pi_{E}}\left(s_{0}\right)-V^{\tilde{\pi}}\left(s_{0}\right)\right|=\left|\theta^{T}\left(\mu^{\pi} E\left(s_{0}\right)-\mu^{\tilde{\pi}}\left(s_{0}\right)\right)\right| \leq\left\|\mu^{\pi} E\left(s_{0}\right)-\mu^{\tilde{\pi}}\left(s_{0}\right)\right\|_{2}
$$


This equation uses the Cauchy-Schwarz inequality and the assumption that $\|\theta\|_{2} \leq 1$. Therefore, the approach described here does not ensure to retrieve the true reward function $R^{*}$, but to act as well as the expert under this reward function (and actually under any reward function).

Let us now describe the algorithm proposed in [Abbeel and $\mathrm{Ng}, 2004$ ] to achieve this goal:

1. Starts with some initial policy $\pi^{(0)}$ and compute $\mu^{\pi^{(0)}}\left(s_{0}\right)$. Set $j=1$;

2. Compute $t^{(j)}=\max _{\theta:\|\theta\|_{2} \leq 1} \min _{k \in\{0, j-1\}} \theta^{T}\left(\mu^{\pi_{E}}\left(s_{0}\right)-\mu^{\pi^{(k)}}\left(s_{0}\right)\right)$ and let $\theta^{(j)}$ be the value attaining this maximum. At this step, one searches for the reward function which maximizes the distance between the value of the expert at $s_{0}$ and the value of any policy computed so far (still at $s_{0}$ ). This optimization problem can be solved using a quadratic programming approach or a projection algorithm [Abbeel and $\mathrm{Ng}, 2004$ ];

3. if $t^{(j)} \leq \epsilon$, terminate. The algorithm outputs a set of policies $\left\{\pi^{(0)}, \ldots, \pi^{(j-1)}\right\}$ among which the user chooses manually or automatically the closest to the expert (see [Abbeel and $\mathrm{Ng}, 2004$ ] for details on how to choose this policy). Notice that the last policy is not necessarily the best (as illustrated in Section 4);

4. solve the MDP with the reward function $R^{(j)}(s)=\left(\theta^{(j)}\right)^{T} \phi(s)$ and denote $\pi^{(j)}$ the associated optimal policy. Compute $\mu^{\pi^{(j)}}\left(s_{0}\right)$;

5. set $j \leftarrow j+1$ and go back to step 2 .

There remain three problems: computing the feature expectation of the expert, solving the MDP and computing feature expectations of intermediate policies.

As suggested in [Abbeel and Ng, 2004], solving the MDP can be done approximately by using any appropriate reinforcement learning algorithm. In this paper, we use the Least-Squares Policy Iteration (LSPI) algorithm [Lagoudakis and Parr, 2003]. There remains to estimate feature expectations. In [Abbeel and $\mathrm{Ng}, 2004], \mu^{\pi_{E}}\left(s_{0}\right)$ is estimated using a Monte Carlo approach over $m$ trajectories: $\hat{\mu}_{E}\left(s_{0}\right)=\frac{1}{m} \sum_{h=1}^{m} \sum_{t=0}^{\infty} \gamma^{t} \phi\left(s_{t}^{(h)}\right)$ This approach does not hold if only transitions of the expert are available, or if trajectories are too long (in this case, it is still possible to truncate them). For intermediate policies, it is also suggested to estimate associated feature expectations using a Monte Carlo approach (if they cannot be computed exactly). This is more constraining than for the expert, as this assumes that a simulator of the system is available. In order to address these problems, we introduce a temporal-difference-based algorithm to estimate these feature expectations.

\section{$3 \quad$ LSTD- $\mu$}

Let us write the definition of the $i^{\text {th }}$ component of a feature expectation $\mu^{\pi}(s)$ for some policy $\pi: \mu_{i}^{\pi}(s)=E\left[\sum_{t=0}^{\infty} \gamma^{t} \phi_{i}\left(s_{t}\right) \mid s_{0}=s, \pi\right]$ This is exactly the definition 
of the value function of the policy $\pi$ for the MDP considered with the $i^{\text {th }}$ basis function $\phi_{i}(s)$ as the reward function. There exist many algorithms to estimate a value function, any of them can be used to estimate $\mu_{i}^{\pi}$. Based on this remark, we propose to use specifically the least-squares temporal difference (LSTD) algorithm [Bradtke and Barto, 1996] to estimate each component of the feature expectation (as each of these components can be understood as a value function related to a specific and known reward function).

Assume that a set of transitions $\left\{\left(s_{t}, r_{t}, s_{t+1}\right)_{1 \leq t \leq n}\right\}$ sampled according to the policy $\pi$ is available. We assume that value functions are searched for in some hypothesis space $\mathcal{H}_{\psi}=\left\{\hat{V}_{\xi}(s)=\sum_{i=1}^{q} \xi_{i} \psi_{i}(s)=\xi^{T} \psi(s), \xi \in \mathbb{R}^{q}\right\}$. As reward and value functions are possibly quite different, another hypothesis space is considered for value function estimation. But if $\mathcal{H}_{\phi}$ is rich enough, one can still consider $\mathcal{H}_{\psi}=\mathcal{H}_{\phi}$. Therefore, we are looking for an approximation of the following form: $\hat{\mu}_{i}^{\pi}(s)=\left(\xi_{i}^{*}\right)^{T} \psi(s)$ The parameter vector $\xi_{i}^{*}$ is here the LSTD estimate:

$$
\xi_{i}^{*}=\left(\sum_{t=1}^{n} \psi\left(s_{t}\right)\left(\psi\left(s_{t}\right)-\gamma \psi\left(s_{t}^{\prime}\right)\right)^{T}\right)^{-1} \sum_{t=1}^{n} \psi\left(s_{t}\right) \phi_{i}\left(s_{t}\right)
$$

For apprenticeship learning, we are interested more particularly in $\hat{\mu}^{\pi}\left(s_{0}\right)$. Let $\Psi=\left(\psi_{i}\left(s_{t}\right)\right)_{t, i}$ be the $n \times q$ matrix of values predictors, $\Delta \Psi=\left(\psi_{i}\left(s_{t}\right)-\gamma \psi_{i}\left(s_{t}^{\prime}\right)\right)_{t, i}$ be the related $n \times q$ matrix and $\Phi=\left(\phi_{i}\left(s_{t}\right)\right)_{t, i}$ the $n \times p$ matrix of reward predictors. It can be easily checked that $\hat{\mu}^{\pi}\left(s_{0}\right)$ satisfies:

$$
\left(\hat{\mu}^{\pi}\left(s_{0}\right)\right)^{T}=\psi\left(s_{0}\right)^{T}\left(\Psi^{T} \Delta \Psi\right)^{-1} \Psi^{T} \Phi
$$

This provides an efficient way to estimate the feature expectation of the expert in $s_{0}$.

There remains to compute the feature expectations of intermediate policies, which should be done in an off-policy manner (that is without explicitly sampling trajectories according to the policy of interest). To do so, still interpreting each component of the feature expectation as a value function, we introduce a stateaction feature expectation defined as follows (much as the classic $Q$-function extends the value function): $\mu^{\pi}(s, a)=E\left[\sum_{t=0}^{\infty} \gamma^{t} \phi\left(s_{t}\right) \mid s_{0}=s, a_{0}=a, \pi\right] \mathrm{Com}$ pared to the classic feature expectation, this definition adds a degree of freedom on the first action to be chosen before following the policy $\pi$. With a slightly different definition of the related hypothesis space, each component of this feature expectation can still be estimated using the LSTD algorithm (namely using the LSTD-Q algorithm [Lagoudakis and Parr, 2003]). The clear advantage of introducing the state-action feature expectation is that this additional degree of freedom allows off-policy learning. Extending LSTD- $\mu$ to state-action LSTD- $\mu$ is done in the same manner as LSTD is extended to LSTD-Q [Lagoudakis and Parr, 2003], technical details are not provided here for the clarity of exposition.

Given the (state-action) LSTD- $\mu$ algorithm, the apprenticeship learning algorithm presented in Section 2 can be easily extended to a batch and off-policy 
setting. The solely available data is a set of transitions sampled according to the expert policy (and possibly a set of sub-optimal trajectories). The corresponding feature expectation for the starting state $s_{0}$ is estimated with the LSTD- $\mu$ algorithm. At step 4 of this algorithm, the MDP is (approximately) solved using LSPI [Lagoudakis and Parr, 2003] (an approximate policy iteration algorithm using LSTD-Q as the off-policy $Q$-function estimator). The corresponding feature expectation at state $s_{0}$ is estimated using the proposed state-action LSTD- $\mu$.

Before presenting some experimental results, let us stress that LSTD- $\mu$ is simply the LSTD algorithm applied to a specific reward function. Although quite clear, the idea of using a temporal difference algorithm to estimate the feature expectation is new, as far as we know. A clear advantage of the proposed approach is that any theoretical result holding for LSTD also holds for LSTD- $\mu$, such as convergence [Nedić and Bertsekas, 2003] or finite-sample [Lazaric et al., 2010] analysis for example.

\section{Experimental benchmark}

This section provides experimental results on two complementary problems. Subsection 4.1 details the protocol and the results while subsection 4.2 inspects the meaning of the different quality criteria.

\subsection{Experiment description and results}

GridWorld The first experimental benchmark chosen here is one of those proposed in [Ng and Russell, 2000], a 5x5 grid world. The agent is in one of the cells of the grid (whose coordinates is the state) and can choose at each step one of the four compass directions (the action). With probability 0.9 , the agent moves in the intended direction. With probability 0.1 , the direction is randomly drawn. The reward optimized by the expert is 0 everywhere except in the upper-right cell, where it is 1 . For every policy, an episode ends when the upper right cell is attained, or after 20 steps. At the start of each episode, the agent is in the lower-left corner of the grid (the opposite of where the reward is). Both the state and action spaces are finite and of small cardinality. Hence, the chosen feature functions $\phi$ and $\psi$ are the typical features of a tabular representation: 0 everywhere except for the component corresponding to the current state (-action pair).

Both Abbeel and Ng [2004]'s algorithm (from now on referred to as the MC variant) and our adaptation (referred to as the LSTD variant) are tested side by side. The MDP solver of the MC variant is LSPI with a sample source that covers the whole grid (each state has a mean visitation count of more than 150) and draws its action randomly. Both $\mu^{\pi^{(j)}}\left(s_{0}\right)$ and $\mu^{\pi_{E}}\left(s_{0}\right)$ are computed thanks to a Monte-Carlo estimation with enough samples to make the variance negligible. We consider both these computations as perfect theoretical solvers for all intended purpose on this toy problem. We thus are in the case intended by Abbeel and $\mathrm{Ng}$ [2004]. On the other hand the LSTD variant is used without accessing 


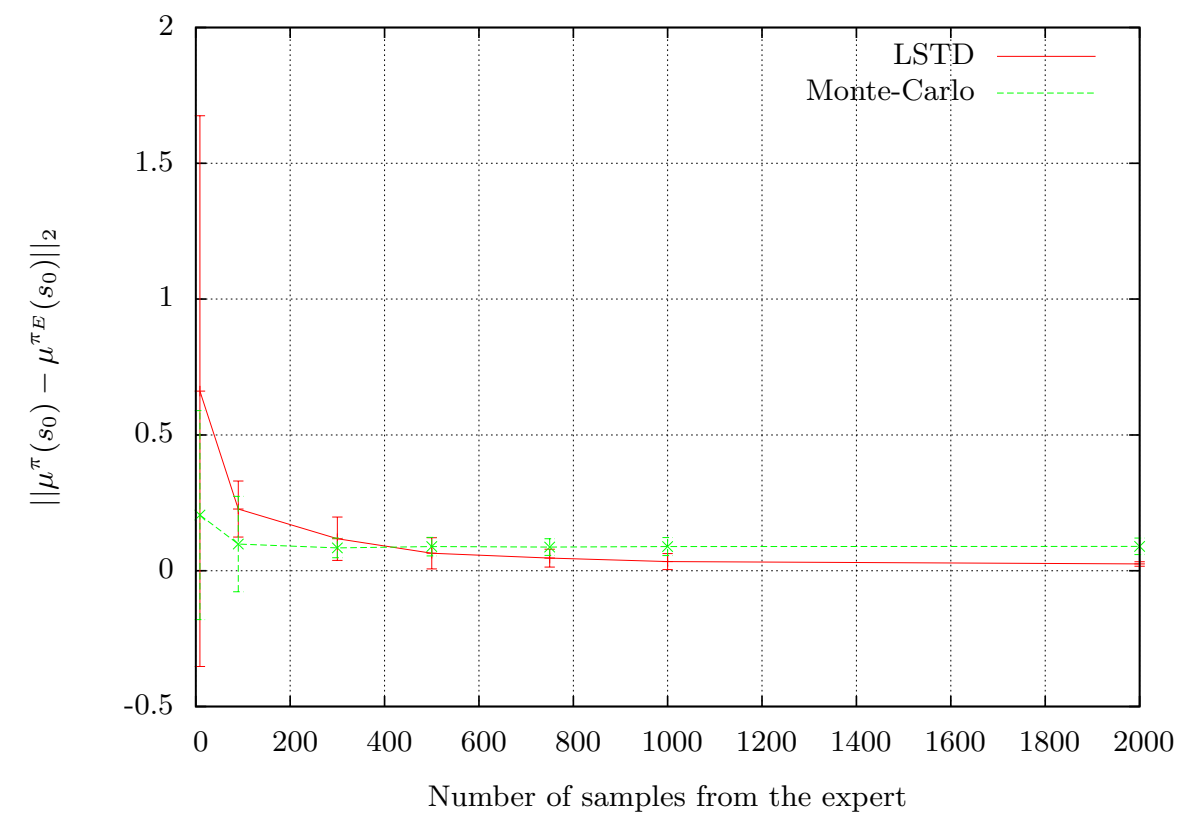

Fig. 1. $\left\|\mu^{\pi}\left(s_{0}\right)-\mu^{\pi_{E}}\left(s_{0}\right)\right\|_{2}$ with respect to the number of samples available from the expert. The error bars represent the standard deviation over 100 runs. 
a simulator. It uses LSPI and LSTD- $\mu$, fed only with the expert's transitions (although we could also use non expert transitions to compute intermediate policies, if available). This corresponds to a real-life setting where data generation is expensive and the system cannot be controlled by an untrained machine.

We want to compare the efficiency of both versions of the algorithm with respect to the number of samples available from the expert, as these samples usually are the bottleneck. Indeed as they are quite costly (in both means and human time) to generate they are often not in abundance hence the critical need for an algorithm to be expert-sample efficient. Having a simulator at one's disposal can also be difficult. For the simple problem we use this is however not an issue. The discussion about the choice of the performance metric has its own dedicated subsection (subsection 4.2). We use here the $\left\|\mu^{\pi^{(j)}}\left(s_{0}\right)-\mu^{\pi_{E}}\left(s_{0}\right)\right\|_{2}$ error term. Fig. 1 shows, for some numbers of samples from the expert, the value of $\left\|\mu^{\pi^{(j)}}\left(s_{0}\right)-\mu^{\pi_{E}}\left(s_{0}\right)\right\|_{2}$ after the algorithm converged or attained the maximum number of iterations (we used 40). The best policy is found by LSTD variant after one iteration only ${ }^{1}$ whereas in the $\mathrm{MC}$ variant, convergence happens after at least ten iterations. The best policy is not always the last, and although it has always been observed so far with the LSTD variant, there is absolutely no way to tell whether this is a feature. The score of the best policy (not the last) according to the $\left\|\mu^{\pi^{(j)}}\left(s_{0}\right)-\mu^{\pi_{E}}\left(s_{0}\right)\right\|_{2}$ error term is plotted here. We can see that although the LSTD variant is not as good as the MC variant when very few samples are available, both algorithms quickly converge to almost the same value; our version converges to a slightly lower error value. The fact that our variant can work in a batch, off-policy and model-free setting should make it suitable to a range of tasks inaccessible to the $\mathrm{MC}$ variant, the requirement of a simulator being often constraining.

Inverted Pendulum Another classic toy problem is the inverted pendulum, used for example in [Lagoudakis and Parr, 2003] from where we drew the exact configuration (mass of the pendulum, length of the arm, definition of the feature space, etc.). In this setting, the machine must imitate an expert who maintains the unstable equilibrium of an inverted pendulum allowed to move along only one axis. A balancing step is considered successful (reward 0) when the angle is less than $\frac{\pi}{2}$. It is considered a failure (reward -1 ) when the pendulum has fallen (i.e. the angle exceeds $\frac{\pi}{2}$ ). A run is stopped after a failure or after 3000 successful balancing steps.

This problem bears two fundamuntal differences with the previous one and thus comes as complementary to it. First, it presents a continuous state space whereas in the GridWorld a tabular representation was possible. Then, the initial state is randomly drawn from a non singleton subspace of the state space.

\footnotetext{
${ }^{1}$ Precise reasons why it happens are not clear now, but certainly have something to do with the fact that all the estimations are made along the same distribution of samples.
} 


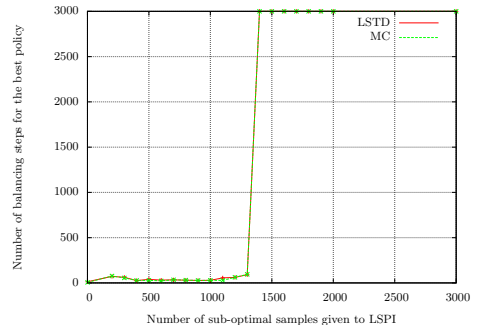

Fig. 2. Number of balancing steps for the policies found by both variants with respect to the number of samples from a sub-optimal policy given to LSPI. Note the absence of middle ground in the quality.



Fig. 3. Same plot as Fig. 2, with mean and standard deviation over 100 runs. The big standard deviation stems from the absence of middle ground in the quality of the policies. The increasing mean w.r.t. the abscissa means that the proportion of good policies increases with the number of samples from a sub-optimal policy given to LSPI.

This last point is addressed differently by the LSTD variant and the MC variant. the $\mathrm{MC}$ variant will naturally sample the initial state distribution at each new trajectory from the expert. The LSTD variant, on the other hand, still does not need complete trajectories from the expert but mere samples. As it approximates the whole $\mu^{\pi}$ function and not just $\mu^{\pi}\left(s_{0}\right)$, it is possible to compute the mean of the approximation $\hat{\mu}^{\pi}$ over a few states drawn with the same distribution as the initial states.

A striking finding on this toy problem is that the parameter controlling the success or the failure of the experiment is not the number of samples from the expert, but the quality of the samples available to LSPI to solve the MDP. $A$ contrario to what happened in the previous toy problem, there are fewer samples from the expert than what LSPI needs to solve the MDP as they do not cover the whole state space. When given only these samples, the LSTD variant fails. The MC variant, however, is not bound by such restrictions and can use as many samples as it needs from a simulator. Thus, with only one trajectory from the expert (100 samples) the MC variant is able to successfully balance the pendulum for more than 3000 steps. The problem here stems from the fact that we don't learn the reward, but a policy that maximizes the reward. If the optimal control problem was solved separately, learning the reward only from the samples of the expert would be possible.

The sensitivity of both algorithms to the number of samples available to LSPI is extreme, as is shown in Fig. 2. It may seem nonsensical to restrict the number 
of samples available to LSPI for the MC variant as it can access a simulator, but this has been done to show that both variants exhibit the same behavior, hence allowing us to locate the source of this behavior in what both algorithms have in common (the use of LSPI inside the structure of [Abbeel and Ng, 2004]) excluding the point where they differ (LSTD- $\mu$ and Monte-Carlo).

Fig. 3 shows that when given samples from a sub-optimal policy, the LSTD variant can sort the problem out, statistically almost always with 3000 suboptimal samples, and sometimes with as low as 200 sub-optimal samples. Such a setting is still batch, off-policy and model-free. When given enough sub-optimal samples, both variants are successful with only one trajectory from the expert (i.e. 100 samples, this is what is plotted here). Giving more trajectories from the expert does not improve the success rate.

\subsection{Discussion about the quality criterion}



Fig. 4. Different criteria with respect to the number of iterations for a run of the MC variant.

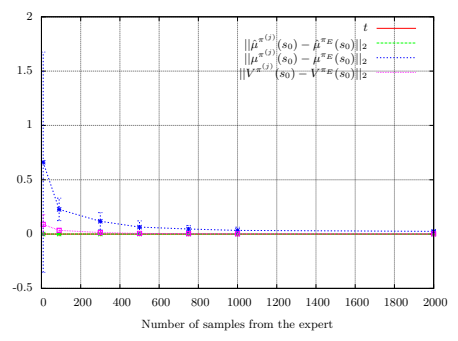

Fig. 5. Different criteria with respect to the number of samples from the expert, for several runs of the LSTD variant. We can see that the algorithm is blind, as all it has access to is always zero. The true error values, however, smoothly converge to something small. Knowing how many expert samples are actually needed in a real world problem is an open question. The error bars represent the standard deviation over 100 runs.

Fig. 4 and 5 illustrate (using the GridWorld problem) the difficulty of choosing the quality criterion ; Fig. 4 shows four different quality criteria during a run of the MC variant. Fig. 5 shows the same criteria for several runs of the LSTD variant with a varying number of samples from the expert. The 
$\left\|\mu^{\pi^{(j)}}\left(s_{0}\right)-\mu^{\pi_{E}}\left(s_{0}\right)\right\|_{2}$ term is widely discussed in [Abbeel and Ng, 2004]'s additional material. It bears an interesting relation with the difference between the expert's value function and the current value function in the initial state with respect to the current reward (Eq. 3).

The fact that the curves of the true error term $\left\|\mu^{\pi^{(j)}}\left(s_{0}\right)-\mu^{\pi_{E}}\left(s_{0}\right)\right\|_{2}$ and its estimate $\left\|\hat{\mu}^{\pi^{(j)}}\left(s_{0}\right)-\hat{\mu}^{\pi_{E}}\left(s_{0}\right)\right\|_{2}$ are indistinguishable in Fig. 4 means that, for it has access to a cheap simulator, the MC variant works as if it had access to the exact values. This however cannot be said of the LSTD variant, for which the two curves are indeed different (Fig. 5). Not knowing the true value of $\mu^{\pi_{E}}\left(s_{0}\right)$ may be a problem for our variant, as it can introduce an error in the stopping criterion of the algorithm.

It shall be noted that although it plays its role, the halt criterion is not a good measure of the quality of the current policy in the MC variant either, as it can be low (and thus halt the algorithm) when the policy is bad. The best policy, however, can be easily chosen among all those created during the execution of the algorithm thanks to the $\left\|\mu^{\pi^{(j)}}\left(s_{0}\right)-\mu^{\pi_{E}}\left(s_{0}\right)\right\|_{2}$ term, which the $\mathrm{MC}$ variant can compute. When this term is low, the objective performance (that is, $V^{\pi_{E}}\left(s_{0}\right)-V^{\pi^{(j)}}\left(s_{0}\right)$ with respect to the unknown true reward function) is low too.

\section{Conclusion}

Given some transitions generated by an expert controlling a system and maximizing in the long run an unknown reward, we ported Abbeel and Ng [2004]'s approach to apprenticeship learning via inverse reinforcement learning to a batch, model-free, off-policy setting. Experimentally, there is a need for either a slightly bigger number of samples from the expert or some samples from a sub-optimal policy. We believe this cost is not prohibitive as our approach only requires isolated samples which are often less difficult to get than whole trajectories as needed by the original approach. Furthermore, tranferring the reward and not the policy may overcome this difficulty. We intend to do this in a real life setting. The simple idea of using LSTD to estimate the feature expectation could be applied to other algorithms as well, for example [Abbeel and Ng, 2004; Syed et al., 2008; Syed and Schapire, 2008; Ziebart et al., 2008]. 


\section{Bibliography}

P. Abbeel and A.Y. Ng. Apprenticeship learning via inverse reinforcement learning. In Proceedings of the twenty-first international conference on Machine learning, page 1. ACM, 2004.

S.J. Bradtke and A.G. Barto. Linear least-squares algorithms for temporal difference learning. Machine Learning, 22(1):33-57, 1996.

J.Z. Kolter, P. Abbeel, and A.Y. Ng. Hierarchical apprenticeship learning with application to quadruped locomotion. In Neural information processing systems, volume 20. Citeseer, 2008.

M.G. Lagoudakis and R. Parr. Least-squares policy iteration. The Journal of Machine Learning Research, 4:1107-1149, 2003.

A. Lazaric, M. Ghavamzadeh, and R. Munos. Finite-sample analysis of lstd. In Proceedings of the 27th International Conference on Machine Learning, 2010.

A. Nedić and DP Bertsekas. Least squares policy evaluation algorithms with linear function approximation. Discrete Event Dynamic Systems, 13(1):79110, 2003.

G. Neu and C. Szepesvári. Apprenticeship learning using inverse reinforcement learning and gradient methods. In Proc. UAI, pages 295-302. Citeseer, 2007.

A.Y. Ng and S. Russell. Algorithms for inverse reinforcement learning. In Proceedings of the Seventeenth International Conference on Machine Learning, pages 663-670. Morgan Kaufmann Publishers Inc., 2000.

D. Ramachandran and E. Amir. Bayesian inverse reinforcement learning. Urbana, 51:61801, 2007.

N.D. Ratliff, J.A. Bagnell, and M.A. Zinkevich. Maximum margin planning. In Proceedings of the 23rd international conference on Machine learning, page 736. ACM, 2006.

N. Ratliff, J.A. Bagnell, and S.S. Srinivasa. Imitation learning for locomotion and manipulation. In Humanoid Robots, 2007 7th IEEE-RAS International Conference on, pages 392-397. IEEE, 2007.

N. Ratliff, D. Bradley, J.A. Bagnell, and J. Chestnutt. Boosting structured prediction for imitation learning. Advances in Neural Information Processing Systems, 19:1153, 2007.

S. Russell. Learning agents for uncertain environments (extended abstract). In Proceedings of the eleventh annual conference on Computational learning theory, page 103. ACM, 1998.

R.S. Sutton and A.G. Barto. Reinforcement learning. MIT Press, 1998.

U. Syed and R.E. Schapire. A game-theoretic approach to apprenticeship learning. Advances in neural information processing systems, 20:1449-1456, 2008.

U. Syed, M. Bowling, and R.E. Schapire. Apprenticeship learning using linear programming. In Proceedings of the 25th international conference on Machine learning, pages 1032-1039. ACM, 2008.

B.D. Ziebart, A. Maas, J.A. Bagnell, and A.K. Dey. Maximum entropy inverse reinforcement learning. In Proc. AAAI, pages 1433-1438, 2008. 